Portland State University

PDXScholar

\title{
Exploring Consumer Psychographics of Ethnic Representation within the Cosmetic Industry
}

Johnny A. Hickman

Portland State University

Follow this and additional works at: https://pdxscholar.library.pdx.edu/honorstheses

Part of the Marketing Commons

Let us know how access to this document benefits you.

\section{Recommended Citation}

Hickman, Johnny A., "Exploring Consumer Psychographics of Ethnic Representation within the Cosmetic Industry" (2020). University Honors Theses. Paper 827.

https://doi.org/10.15760/honors.846

This Thesis is brought to you for free and open access. It has been accepted for inclusion in University Honors Theses by an authorized administrator of PDXScholar. Please contact us if we can make this document more accessible: pdxscholar@pdx.edu. 
Exploring Consumer Psychographics of Ethnic Representation within the Cosmetic Industry

by

Johnny A. Hickman

An undergraduate honors thesis submitted in partial fulfillment of the

requirements for the degree of

Bachelor of Science

in

University Honors 
and

Business

Thesis Adviser

Jacob Suher

Portland State University

2020 


\section{Table of Contents}

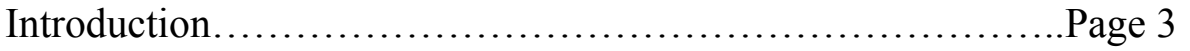

Diversity \& Inclusion within the Cosmetic Industry............Page 4

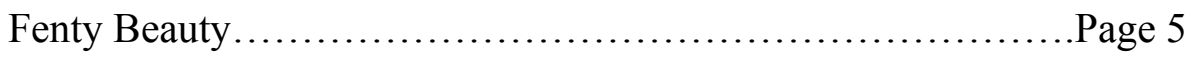

Research Question......................................Page 8

Tarte Cosmetics....................................... 9

Primary Research Results..................................Page 11

Research Analysis.......................................Page 13

Conclusion.................................................... 16

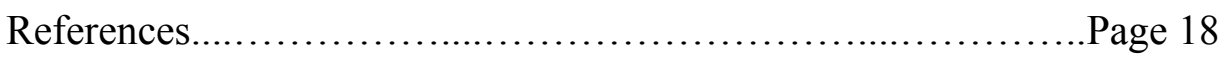

Copy of Survey................................................... 20 


\section{Introduction}

The cosmetics industry dates back as far as 4,000 BC, when Ancient Egyptians wore kohl eyeliner (Avenue Institute). In our current day, the cosmetics industry is worth $\$ 93.5$ billion dollars (Biron, B.), contributes 9.7\% of total e-commerce sales in the U.S. (Duncan, E.) and has an online presence of 22 million followers and counting. The continued success of the cosmetics industry can be attributed to the rapid rise of social media, considering its one of the top topics across all platforms (Clement, J.). There are a plethora of brands that are all made to serve targeted audiences from vegans to the LGBTQ community. Even retailers that previously did not carry cosmetics, such as H\&M and Lululemon are beginning to expand their cosmetics lines to become a part of such a lucrative industry (Biron, B.). 


\section{Diversity \& Inclusion within the Cosmetic Industry}

Diversity and inclusion is a concept that, for a long time has remained just that, a concept. However, in recent years, retailers in all industries are becoming more and more cognizant of the importance of diversity and including diverse figures in their advertising and ensuring that they are also represented in their lines. Nude no longer meant, a soft peach color that would make the ideal nude for a caucasian person, but a wide array of colors that would closely match a person's skin tone. However, this ideal has not been implemented well in the cosmetics industry.

Historically, cosmetic brands carried complexion products, such as foundation and face powder, in which the majority of these shades were better suited for someone with fair to olive skin tones and the least amount are best suited for deeper skin tones. It was not until 2017 when a new brand, founded by a non-traditional founder, revolutionized the Beauty Community's word with a concept that seemed incredibly prudent. Fenty Beauty, created by musician Rihanna, introduced a new approach to complexion products and expanded upon the medium to deep skin tone ranges with 40 shades (Rodulfo, K). This approach caused an uproar in the cosmetics industry and led consumers to question why a new brand in its infancy stage was the first to accomplish this, when there are scores of brands that were much more established that had not made this leap. This level of identity established with consumers created an even deeper connection between brand and consumer. It made consumers feel represented in a way that they had not been before. This seemingly obvious change, made an astonishingly large population feel acknowledged and understood and there was no way that they would be able to accept being forgotten any longer. 


\section{Fenty Beauty}

The year 2017 initiated a wave of change that strived for diversity and inclusion that was trail blazed by the introduction of Fenty Beauty. The first collection contained 40 shades of complexion products that launched in 1,600 stores across 17 countries (Shatzman, C.). The ethos of Fenty Beauty is to get the 'Fenty Face' that promises women of every color a radiant and glowing complexion, just like Rihanna's. While it is incredibly intriguing to be promised to look identical to the icon, the more significant promise is that it is available to women of all complexions, not those that are medium to fair skin tones. This was a great concern that led to the creation of Fenty Beauty, as Fenty Beauty's predecessors previously did not create a shade range that was accommodating to women of deeper skin tones.

Fenty Beauty's mission was clear and simple; "so that women everywhere would be included" (Fenty Beauty). This was incredibly poignant as prior to Fenty Beauty's launch, consumers often had a hard time finding foundation that matched their skin tone and if they did, it typically was not the right consistency (Huffpot). It was not just the average consumer that struggled to find a complexion product that worked for them, world renowned model Chanel Iman often had to mix her own products for the runway as artists typically did not have the products that would match. Fenty Beauty's business model is structured to ensure that it fulfills its commitment to inclusivity. Prior to launching in 2017, Fenty Beauty conducted a two year research and development process to ensure that their formulas are created to accurately match the neglected deep and dark shades (Sharma, T.). In addition to the care that was put into research and development, Fenty Beauty launched during New York fashion week, which capitalized on the publicity that the gathering would already garner. This strategy created a buzz 
around Fenty Beauty that led to several publications reviewing it and thus peaking the interests of consumers (Sharma, T.).

Because Fenty Beauty was able to create such pandemonium around its brand and its mission statement, it immediately entered the market completely differentiated from the rest of its competitors. This level of differentiation not only sparks interest in consumers, but it establishes that there was a need for such an extensive shade range that was not acknowledged prior. This is a crucial differentiator as the majority of Fenty Beauty's competitors on the market had only contained a maximum of 30 shades. The consumer's response was overwhelmingly positive, especially from those who did not previously feel represented by other brands. Although having an icon such as Rihanna not only backing the brand, but leading it, is a large contributing factor into the brand's success; what it's trying to accomplish is what truly resonates with consumers. This is the true differentiator between Fenty Beauty and commercial brands such as Cover Girl, who often feature women of color in their TV and Magazine ads. That level of connection cannot be established, just by show-casing women of various skin tones; it requires a message grounded in the reasoning behind it, otherwise it will not achieve the same type of connection with the consumer base. Having this voice come from such an influential figure that understood the struggle to find a shade that matched their complexion deepened the connection of the brand with consumers and made the brand more trustworthy. The brand was solving a problem that had long existed prior to Rihanna's introduction into popular culture. Competitors of Fenty Beauty can take note of the approach it has taken with its shade range and begin introducing more options into their lines. Although this may run the risk of being perceived as inauthentic, as Fenty Beauty had a successful attempt at this first; it makes 
popular brands that consumers previously were not able to find products that matched them well available to them.

However, competitors must be diligent when executing a strategy such as Fenty Beauty's as it can potentially be perceived as disingenuous to consumers, especially with the approach they may take with advertising campaigns. Considering Fenty Beauty's mission statement was immediately clear to its audience upon launch based on the advertising campaign utilized; competitors must find other ways to convey this message of inclusivity without treading on Fenty Beauty's momentum. This presents a very unique and nuanced challenge for competitors, as they will need to create a strategy that utilizes their branding and tie it in with the message of being inclusive. However, if this was not a strong element of the brand or the brand's mission statement, they will need to find a way to carefully introduce this concept to their consumer base and shift their perception. 


\section{Research Question}

Consumers are more sensitive than ever to a brand that has contrived messaging. Despite brands like Maybeline and Covergirl featuring famous women of color in their ads, there has never been a reaction to their brand like there has been for Fenty Beauty. This is because of Fenty Beauty's authentic mission to fulfill a need for consumers. This is a level of authenticity that cannot be matched by simply show-casing women of various skin tones and proceeding to provide a limited amount of products. Is brand authenticity the key to winning over consumers and significant in consumers' ability to form a connection with a brand? 


\section{Tarte Cosmetics}

The 2000s marked a new era for the cosmetics industry. Packaging was beginning to become more decorative, color options were beginning to expand and companies were attempting to introduce concept kits. Tarte Cosmetics was founded in 1999 by Maureen Kelly and was at the forefront of this renaissance of the cosmetics industry. Tarte Cosmetics is known for its creative and innovative product launches that are typically themed and have garnered a massive following and is now worth $\$ 100.3$ million as of 2019. Tarte Cosmetics has always had its finger on the pulse of the next biggest wave in cosmetics, from their long lasting Amazonian clay formula, to their peach collection that introduced a warm toned palette. However, as innovative as they are when it comes to their ad campaigns and product designs were, there was a significant lack of innovation when it came to their shade ranges. Like most of their competitors, they provided only 30 shades when it came to their complexion products that were of the typical variety as seen by other companies. This was met with outrage from consumers as they were continuously impressed by the level of intricate detail that the companies instilled, that surely such a brand would be privy to the needs of consumers who fall outside of the typical shade range.

Tarte Cosmetics' mission statement is to use 'Good-for-you, naturally derived ingredients' in its products, which was a major differentiator from its competitors upon launching. Prior to launch, there were not many companies that used natural ingredients that would be considered 'prestige' like Tarte Cosmetics. Although an expansion of shade ranges doesn't completely fall in line with their mission statement; it would have been an incredibly lucrative opportunity for Tarte Cosmetics to consider, as it would have been the first brand to not only have an expansive shade range for women of all complexions, but made from natural ingredients as well. This in turn tarnished Tarte Cosmetics' reputation amongst consumers once Fenty Beauty launched its line as it had been considered one of the leading innovative brands available, regardless of what their mission statement was. The same could be said if Adidas created a foundation where a pair of shoes was donated to children in need for every purchase. Consumers 
would be questioning Nike, bewildered as to why they have not been doing this all along as they are considered the number one brand globally.

In comparison to Tarte Cosmetics, Fenty Beauty has garnered a reputable name for itself upon launch. The overall reception of the products from consumers was overwhelmingly positive, as consumers all over the world sung praises in their reviews on social media about the level of inclusivity provided in Fenty Beauty's shade range. In addition to this, the ad campaigns that featured at least 30 women of varying skin tones sparked a positive sentiment from consumers that was seemingly a direct response to previous ad campaigns akin to Tarte Cosmetics. Because of the level of representation in the products themselves and the marketing campaigns, this established a deep connection for consumers that Tarte Cosmetics was failing to achieve. Because Fenty Beauty's launch was so strong in its messaging, beauty for all, it clearly drew a line in the sand that differentiated it from Tarte Cosmetics in a way that made it nearly impossible for Tarte Cosmetics to be able to rebound from.

The difficulty consumers of color face when it comes to finding shade match for something as intimate as a complexion product goes beyond finding a makeup product that looks good, it's an intrinsic need. This need that is created from the lack of options can have negative effects on the consumer's psyche and thus affecting their overall perception of themselves. The same can be said about consumers who are limited to a small amount of clothing stores, due to the majority of retailers only carrying a limited range of sizes. This is a need that can only be fulfilled when the consumer feels included and represented. 


\section{Primary Research Results}

In conducting primary research using a survey, a sample set was obtained of 249 participants. Out of this sample size, 155 participants were between the ages of 18 and 24, 73 participants were between ages of 35 and 44, 18 participants were between the ages of 35 and 44 and three participants were between the ages of 45 and 54.170 participants were female, 65 participants were male, 12 participants preferred not to say and 2 participants identified as 'Other'. 120 participants were White, 50 participants were Asian, 50 participants identified as 'Other', 20 participants were Black, 5 participants were Native Hawaiin/Pacific Islander and 4 participants were American Indian/Alaska Native.

In terms of behavior, 66 participants wear makeup sometimes, 53 participants never wear makeup, 42 participants wear make up half the time and 23 participants always wear makeup. Amongst the participants, 71 participants shop for complexion products sometimes, 57 participants shop for complexion products always, 53 participants never shop for complexion products, 47 participants shop for complexion products most of the time and 21 participants shop for complexion products about half the time. When shopping for complexion products, 81 participants find products that match their skin tone most of the time, 65 find their match sometimes, 39 participants never find a match, 32 participants always find a match and 32 participants find a match about half the time. When looking for a color match, 79 participants spend a moderate amount of time, 76 participants spend a little amount of time, 44 participants

spend none at all, 30 participants spend a lot of time, 20 participants spend a great deal of time. When it comes to mixing products to match their skin tone, 119 participants never mix products, 74 participants sometimes mix products, 22 participants spend most of the time mixing products, 
21 participants spend half the time mixing products and 13 participants always mix products. When viewing a limited selection of shades available for complexion products, 74 participants feel slightly negative, 63 participants feel moderately negative, 54 participants feel extremely negative, 45 participants feel neither positive nor negative, 9 participants feel slightly positive, 3 participants feel moderately positive, 1 participant feels extremely positive. 


\section{Research Analysis}

Because of the sample that I was able to access, this significantly overwhelmed the minority opinion, which is I believe is a key indicator to how larger corporations within the beauty industry need to go out of their way to access the voice of the minority.

As a direct result of the lack of options provided by cosmetic brands, consumers often have to become innovative and find creative ways to make a complexion product suit their skin tone as well as possible. Oftentimes, consumers of deeper skin tones will have to mix together two or more products to create a mixture that matches their skin tone as closely as possible. This is an expensive alternative for consumers who have to purchase multiple items and undoubtedly leads to a loss of sales as only a portion of consumers can afford to continue to purchase multiple complexion products on a regular cadence. However, if consumers were able to only purchase one universal shade that matched their skin tone well, they are more likely to continue to purchase from that same brand for a longer period of time, thus increasing the customer lifetime value and eventually increasing revenue for companies. If shade selection were to expand, 98 participants would feel extremely positive, 69 participants would feel moderately positive, 37 participants would feel neither positive nor negative, 36 participants would feel slightly positive and 3 participants would feel slightly negative, moderately negative and extremely negative. When it comes to representation, 99 participants strongly agree that it is important to them to feel represented by a brand they are purchasing from, 80 participants agreed, 31 participants somewhat agreed, 28 participants neither agreed nor disagreed, 7 participants disagreed and 4 participants somewhat disagreed. 
Black/African American participants make up 6\% of the total participant pool. 6 participants were between the ages of 18 and 24 and 9 participants were between the ages of 25 and $34.36 \%$ of Black participants claimed that they wear makeup most of the time. $33 \%$ of Black participants always shop for complexion products and 33\% of Black participants spend a great deal of time looking for a complexion product that matches their skin tone. $73 \%$ of Black participants feel extremely negative when there is a limited selection of shades available when shopping for complexion products and $73 \%$ of Black participants strongly agree that it is important that they feel represented by the brand that they are purchasing from.

White participants make up $28 \%$ of the total participant pool. 70 White participants are between the ages of 18 and 24, 34 White participants are between the ages of 25 and 34, 13 White participants are between the ages of 35 and 44 and 3 participants are between the ages of 45 and $54.30 \%$ of White participants claimed that they wear makeup most of the time. $26 \%$ of White participants always shop for complexion products and 5\% of White participants spend a great deal of time looking for a complexion product that matches their skin tone. $16 \%$ of White participants feel extremely negative when there is a limited selection of shades available when shopping for complexion products and 35\% of White participants strongly agree that it is important that they feel represented by the brand that they are purchasing from.

In analyzing these two specific groups of participants, it is incredibly clear that the Black participants are much more enthusiastic about makeup as 36\% Black participants wear makeup most of the time, whereas $30 \%$ have indicated this. However, when looking at the amount of time spent looking for a complexion product that is of the right shade, $33 \%$ of Black participants spend a great deal of time, whereas $5 \%$ of White participants spend this amount of time. 
Although these statistics are based off of a modest sample of only 249 participants in the Portland metro area, these results are a reflection of how companies within the cosmetics industry are behaving. There is a significant lack of representation and availability of products available to a demographic that is in need and is arguably more enthusiastic about cosmetics as whole, in comparison to the majority. In order for companies to truly capture the voice of their audience, it is imperative that they take extensive measures to acquire the opinions of the minority audience and begin implementing their findings from said opinions. 


\section{Conclusion}

The lasting negative effects on consumers who feel under-represented are insurmountable. Consumers are always seeking products that will fulfill needs for them and when there is a need as deep as feeling represented, it creates a large gap in the market, especially in a consumer driven market as lucrative as the cosmetic industry. Consumers not only need to feel represented; they crave a connection with a brand. Fenty Beauty is able to fulfill both of these needs, when competitors are typically only able to fulfill one of these needs at a time. This signifies how substantial Fenty Beauty's success is and how it has been able to sustain its sales over the last three years. This is a testament to Fenty Beauty's ability to fulfill such intrinsic needs for consumers; had it not achieved this exceptionally well, its success would not have sustained for this long.

Looking more in-depth into Fenty Beauty's success, it is evident that their mission statement and business model have proven to be what drives its success. This further demonstrates the level of success that Fenty Beauty was able to achieve in such a short period of time and demonstrates the overall positive response from consumers.

In order for the cosmetic industry to continue to make strides in diversity and inclusion and become profitable; there are crucial guidelines that need to be followed. Authenticity is key and consumers are more sensitive than ever to a brand that is contrived. Despite brands like Maybeline and Covergirl featuring famous women of color in their ads, there has never been a reaction to their brand like there has been for Fenty Beauty. This is because of Fenty Beauty's authentic mission to fulfill a need for consumers. It's also important for brands to establish their brand identity early. As Fenty Beauty continues to be innovative in their products, it always 
comes back to their mission statement, so that women everywhere would be included.

Establishing a strong brand identity is key as it differentiates brands from competitors and sends clear messaging to consumers. Accurate representation is vital for brands. Gone are the days where having a few women with deeper complexions in their advertising campaigns would satisfy consumers. It's more than a checkbox now and that same representation needs to be reflected in their product line. Reputable spokesmodels just like Rihanna are vital. In order for consumers to form a deeper connection with a brand, the spokesmodel needs to have a point of connection for consumers, just like Rihanna's first hand experience. Finally, the brand needs to fulfill an intrinsic need for the consumer. With such a large industry, there are plenty of brands that promise a perfect complexion, but only one so far has promised inclusion. Should brands begin to take this matter more seriously, we should start to see more companies embody this business model and more consumers of color feeling included. 


\section{References}

1. A Brief History of The Beauty Industry. (2014, November 26). Retrieved from https://www.avenuefive.edu/brief-history-beauty-industry/

2. Biron, B. (2019, July 9). Beauty has blown up to be a $\$ 532$ billion industry - and analysts say that these 4 trends will make it even bigger. Retrieved from https://www.businessinsider.com/beauty-multibillion-industry-trends-future-2019-7

3. Clement, J. (2019, December 3). Instagram: most-followed beauty brands 2019. Retrieved from https://www.statista.com/statistics/536991/leading-beauty-brands-instagram-followers/

4. Duncan, E. (n.d.). Topic: Cosmetics Industry in the U.S. Retrieved from https://www.statista.com/topics/1008/cosmetics-industry/

5. Fenty Beauty by Rihanna. (n.d.). Retrieved from

6. https://www.lvmh.com/houses/perfumes-cosmetics/fenty-beauty-by-rihanna/

7. Fletcher, D. (2003). Reaching The Ethnic Consumer: A Challenge For Marketers. News International Newspapers Limited, 2-16. Doi:media-diversity.org

8. Lifestyle, Y. (2018, April 9). Rihanna's Humanity - And Not Her Celebrity - Is Why She's Changing The Beauty Industry. Retrieved from https://www.huffpost.com/entry/rihannas-humanity-and-not-her-celebrity-is-why-shes-ch anging-the-beauty-industry_n_5acb7bd7e4b09d0a1195e230

9. Papista, E., \& Dimitriadis, S. (2012). Exploring consumer-brand relationship quality and identification. Qualitative Market Research: An International Journal, 15(1), 33-56. doi:10.1108/13522751211191982 
10. Rodulfo, K. (2019, January 4). Fenty Beauty Is Launching 50 Shades of Concealer.

Retrieved from

https://www.elle.com/beauty/makeup-skin-care/a25725136/rihanna-launch-fenty-beautyconcealer/

11. Sharma, T. (2019, November 3). THE FENTY IMPACT: WHAT BEAUTY MARKETING CAN LEARN FROM RIHANNA. Retrieved from https://medium.com/@tanvi00sharma/the-fenty-impact-what-beauty-marketing-can-learn -from-rihanna-9f12dea11c40

12. Shatzman, C. (2017, September 8). Rihanna's Makeup Line, Fenty Beauty, Makes Its Debut. Retrieved from https://www.forbes.com/sites/celiashatzman/2017/09/08/rihannas-makeup-line-fenty-bea uty-makes-its-debut/\#4849e43c2113

13. Sierra, J. J., Hyman, M. R., \& Torres, I. M. (2009). Using a Models Apparent Ethnicity to Influence Viewer Responses to Print Ads: A Social Identity Theory Perspective. Journal of Current Issues \& Research in Advertising, 31(2), 41-66.

doi:10.1080/10641734.2009.10505265 


\title{
HON 403 Thesis Survey
}

\author{
Start of Block: Default Question Block
}

Q29 Please click >> below to begin the survey.

Q26

Consent to Participate in Research

Introduction

You are being asked to participate in a research study that is being done by Jacob Suher, who is the Principal Investigator, from the Department of Marketing, at Portland State University in Portland, Oregon. This research is studying how consumers evaluate cosmetics. You are being asked to participate in this study because you are a consumer. This form will explain the research study, and will also explain the possible risks as well as the possible benefits to you. We encourage you to talk with your family and friends before you decide to take part in this research study. We recommend printing this consent form for your records. If you have any questions, please ask one of the study investigators. What will happen if I decide to participate?

If you agree to participate, the following things will happen: You will be asked to answer questions about cosmetics.

How long will I be in this study? Participation in this study will take a total of 5 minutes.

What are the risks or side effects of being in this study? There are risks of stress, emotional distress, inconvenience and possible loss of privacy and confidentiality associated with participating in a research study. For more information about risks and discomforts, ask the investigator. What are the benefits to being in this study? There are no direct benefits to participating in this study.

What are the alternatives to being in this study? You are permitted to decline to participate in this survey. How will my information be kept confidential? We will take measures to protect the security of all your personal information, but we cannot guarantee confidentiality of all study data. You will not be asked to provide any personal information or information that could link you with your responses. Information contained in your study records is used by study staff. The 
Portland State University Institutional Review Board (IRB) that oversees human subject research and/or other entities may be permitted to access your records, and there may be times when we are required by law to share your information. It is the investigator's legal obligation to report child abuse, child neglect, elder abuse, harm to self or others or any life-threatening situation to the appropriate authorities, and; therefore, your confidentiality will not be maintained. Your name will not be used in any published reports about this study.

Will I be paid for taking part in this study?

No, payment will not be provided.

Can I stop being in the study once I begin?

Your participation in this study is completely voluntary. You have the right to choose not to participate or to withdraw your participation at any point in this study without penalty or loss of benefits to which you are otherwise entitled. Whom can I call with questions or complaints about this study?

If you have any questions, concerns or complaints at any time about the research study, Jacob Suher, or his associates will be glad to answer them at 503-725-9875. If you need to contact someone after business hours or on weekends, please call 503-577-8974 and ask for Jacob Suher.

Whom can I call with questions about my rights as a research participant? If you have questions regarding your rights as a research participant, you may call the PSU Office for Research Integrity at (503) $725-2227$ or 1(877) $480-4400$. The ORI is the office that supports the PSU Institutional Review Board (IRB). The IRB is a group of people from PSU and the community who provide independent oversight of safety and ethical issues related to research involving human participants. For more information, you may also access the IRB website at https://sites.google.com/a/pdx.edu/research/integrity. CONSENT You are making a decision whether to participate in this study. By completing the survey you are agreeing to participate in this study.

\section{Page Break}

Q27 Instructions:

Please answer all questions to the best of your ability and keep your own personal consumer experience in mind. 
Q17 How likely are you to purchase products from the following display?

Extremely likely (1)

Moderately likely (2)

Slightly likely (3)

Neither likely nor unlikely (4)

Slightly unlikely (5)

Moderately unlikely (6)

Extremely unlikely (7)

\section{End of Block: Display 1}

Start of Block: Display 2

Q16 How likely are you to purchase products from the following display? 
Extremely likely (1)

Moderately likely (2)

Slightly likely (3)

Neither likely nor unlikely (4)

Slightly unlikely (5)

Moderately unlikely (6)

Extremely unlikely (7)

\section{End of Block: Display 2}

Start of Block: Ad 1

Q18 How do you feel viewing the following ad campaign?

Extremely happy (1)

Moderately happy (2)

Slightly happy (3)

Neither happy nor unhappy (4) 
Slightly unhappy (5)

Moderately unhappy (6)

Extremely unhappy (7)

\section{End of Block: Ad 1}

Start of Block: Ad 2

Q19 How do you feel when viewing the following ad campaign?

Extremely happy $(1)$

Moderately happy (2)

Slightly happy (3)

Neither happy nor unhappy (4)

Slightly unhappy (5)

Moderately unhappy (6)

Extremely unhappy (7)

\section{End of Block: Ad 2}


Q12 How do you feel when shopping for complexion products?

Extremely positive (1)

Moderately positive (2)

Slightly positive (3)

Neither positive nor negative (4)

Slightly negative (5)

Moderately negative (6)

Extremely negative (7)

Q13 How do you feel when there is a limited selection of shades available when shopping for complexion products?

Extremely positive (1)

Moderately positive (2)

Slightly positive (3)

Neither positive nor negative (4) 
Slightly negative (5)

Moderately negative (6)

Extremely negative (7)

Q14 How would you feel if the selection of shades by your favorite brand were expanded?

Extremely positive (1)

Moderately positive (2)

Slightly positive (3)

Neither positive nor negative (4)

Slightly negative (5)

Moderately negative (6)

Extremely negative (7)

Q22 It is important that you feel represented by the brand that you are purchasing from?

Strongly agree (1) 
Agree (2)

Somewhat agree (3)

Neither agree nor disagree (4)

Somewhat disagree (5)

Disagree (6)

Strongly disagree (7)

Q15 How would you feel if the selection of shades by your favorite brand were reduced?

Extremely positive (1)

Moderately positive (2)

Slightly positive (3)

Neither positive nor negative (4)

Slightly negative (5)

Moderately negative (6)

Extremely negative (7) 
Q25 How do you feel when you purchase a complexion product that matches your skin tone?

Extremely happy

Moderately happy

Slightly happy (3)

Neither happy nor unhappy (4)

Slightly unhappy (5)

Moderately unhappy (6)

Extremely unhappy (7)

\section{End of Block: How do you feel}

Q23 How likely are you to purchase from a brand that you feel represents you?

Extremely likely (1)

Moderately likely (2)

Slightly likely (3) 
Neither likely nor unlikely (4)

Slightly unlikely (5)

Moderately unlikely (6)

Extremely unlikely (7)

Q24

Please indicate your agreement with the following statement:You would wear complexion products more often if you could easily find the products that match your skin tone.

Strongly agree (1)

Agree (2)

Somewhat agree (3)

Neither agree nor disagree (4)

Somewhat disagree (5)

Disagree (6)

Strongly disagree (7) 


\section{Start of Block: Behavior Qs}

Q5 Do you wear makeup?

Always (1)

Most of the time (2)

About half the time (3)

Sometimes (4)

Never (5)

Q6 What makeup brands are you familiar with? Select all that apply.

MAC Cosmetics (1)

Fenty Beauty (2)

Tarte Cosmetics (3)

Drugstore (Maybelline, Covergirl, Revlon, etc.) (4)

Too Face Cosmetics (5)

Estee Lauder (6) 


\section{Clinique (7)}

Urban Decay Cosmetics (8)

Sephora Collection (9)

Colour Pop Cosmetics (10)

Q7 Do you shop for complexion products such as foundation, concealer and/or face powder?

Always (1)

Most of the time (2)

About half the time (3)

Sometimes (4)

Never (5)

Q11 How many stores do you visit to find the complexion product that best matches your skin tone?

$0-1$ (1)

$1-2(2)$ 

$2-3(11)$
$3-4(3)$
$4-5(4)$
$5+(5)$

Q8 When shopping for complexion products, how often do you find products that match your skin tone?

Always (1)

Most of the time (2)

About half the time (3)

Sometimes (4)

Never (5)

Q9 On average, how much time do you spend looking for your color match for complexion products?

A great deal (1) 
A lot (2)

A moderate amount (3)

A little (4)

None at all (5)

Q10 Do you have to mix products to find the shade that best fits your skin tone?

Always (1)

Most of the time (2)

About half the time (3)

Sometimes (4)

Never (5)

End of Block: Behavior Qs

Start of Block: Demographic Qs

Q1 What is your age?

Under 18 (1)

$18-24(2)$ 
$25-34(3)$

35 - 44 (4)

$45-54(5)$

$55-64(6)$

$65-74(7)$

$75-84(8)$

85 or older (9)

Q2 What is your gender?

Male (1)

Female (2)

Prefer not to say (3)

Other (4)

Q3 What is your ethnicity?

White (1) 
Black or African American (2)

American Indian or Alaska Native (3)

Asian (4)

Native Hawaiian or Pacific Islander (5)

Other (6)

Q4 How would you describe your skin tone?

Fair (1)

Light (2)

Medium (3)

Dark (4)

Deep (5)

\section{End of Block: Demographic Qs}

Start of Block: Thank you

Q28 Thank you for participating in this study!

\section{End of Block: Thank you}

